\title{
Ultrafast all-optical switching of dual-band plasmon- induced transparency in terahertz metamaterials
}

\author{
Hao Sun (孙 豪) $)^{1, \dagger}$, Jianghua Zhang (张江华 $)^{2, \dagger}$, Yuhua Tang (唐玉华 $)^{1, \dagger}$, Hengzhu Liu (刘衡竹) ${ }^{1}$, Jie Yang (杨 杰) $)^{2}$, and \\ Xin Zheng (郑 金金) ${ }^{2^{*}}$ \\ ${ }^{1}$ College of Computer, National University of Defense Technology, Changsha 410073, China \\ ${ }^{2}$ National Innovation Institute of Defense Technology, Beijing 100010, China
}

*Corresponding author: zhengxin@nudt.edu.cn

Received May 18, 2021 | Accepted August 2, 2021 | Posted Online September 28, 2021

\begin{abstract}
An active ultrafast formation and modulation of dual-band plasmon-induced transparency (PIT) effect is theoretically and experimentally studied in a novel metaphotonic device operating in the terahertz regime, for the first time, to the best of our knowledge. Specifically, we designed and fabricated a triatomic metamaterial hybridized with silicon islands following a newly proposed modulating mechanism. In this mechanism, a localized surface plasmon resonance is induced by the broken symmetry of a $\mathrm{C}_{2}$ structure, acting as the quasi-dark mode. Excited by exterior laser pumps, the photo-induced carriers in silicon promote the quasi-dark mode, which shields the near-field coupling between the dark mode and bright mode supported by the triatomic metamaterial, leading to the dynamical modulation of terahertz waves from individual-band into dual-band PIT effects, with a decay constant of 493 ps. Moreover, a remarkable slow light effect occurs in the modulating process, accompanied by the dual-transparent windows. The dynamical switching technique of the dual-band PIT effect introduced in this work highlights the potential usefulness of this metaphotonic device in optical information processing and communication, including multi-frequency filtering, tunable sensors, and optical storage.
\end{abstract}

Keywords: all-optical switching; terahertz metamaterials; dual-band plasmon-induced transparency; ultrafast modulation. DOI: 10.3788/COL202220.013701

\section{Introduction}

The electromagnetically induced transparency (EIT) effect, causing the appearance of a sharp transparent peak in the broad absorption or reflection spectrum, is attracting considerable research attention due to the self-enhanced nonlinearity, refractive index, and ultraslow group velocity. However, the application of the EIT effect is constrained by the extreme quantum condition. The analogue of EIT effect may be applied to other optical scales, such as the terahertz $(\mathrm{THz})$ regime $^{[1-12]}$. Considering that no natural material would interact with electromagnetic field in this region, metamaterials, periodically arranged metal structures, are introduced and employed to control the transmission of $\mathrm{THz}$ waves ${ }^{[13-26]}$. Therefore, if the classical analogue of EIT, that is, plasmon-induced transparency (PIT), can be controlled using metamaterials, the exotic properties of the EIT effect, like reduced group velocity and strong dispersion, can be utilized for applications including slow light devices $^{[27]}$, biochemical sensors ${ }^{[28]}$, and optical buffers ${ }^{[29-31]}$. In previous works, the PIT effect in metamaterials is frequently achieved by utilizing destructive interference between bright modes and dark modes ${ }^{[32]}$. Compared to bright modes, which would naturally couple with free space electromagnetic fields, dark modes are excited by near-field coupling with the bright modes, exhibiting a narrower absorption ${ }^{[33]}$. Importantly, the plasmonic split-ring resonators (SRRs), closed-ring resonators (CRRs), and cut wire (CW) resonators are several meta-atom building blocks that can support bright modes and dark modes $^{[34-36]}$.

More recent attention has focused on the multi-band PIT effect in $\mathrm{THz}$ metamaterials, with multiple transparent windows induced by the coupling effect between $\mathrm{THz}$ resonators ${ }^{[37-39]}$. With regard to the switching of the multi-band PIT effect, the quasi-dark resonator is introduced to weakly couple with the free space electromagnetic fields ${ }^{[40,41]}$. Thus, the multi-band PIT effect occurs, stemming from the near-field electrical coupling between the resonant modes in a three-level system comprising the bright mode, dark mode, and quasi-dark mode ${ }^{[42]}$. An abundance of metamaterial configurations have been exploited to support resonating modes and then make them switch to the multi-band PIT effect ${ }^{[43-46]}$. Hu et al. reported a dual-band EIT-like effect in a metamaterial device that contains the double SRRs and two finite metal strips ${ }^{[43]}$. Tang et al. observed two transparent windows with more than $90 \%$ transmission in 
metamaterials consisting of the square patch and closed ring ${ }^{[44]}$. Dong et al. investigated the polarization-independent dual-band PIT effect at a mid-infrared frequency in a metal-graphene device, with the structure composed of two types of center symmetric meta-atoms ${ }^{[45]}$. Kim et al. numerically studied the electromagnetic response of the multi-band EIT-like effect in a dual-ring metamaterial ${ }^{[46]}$. Most researchers focus on the static modulation of multi-band PIT effect. Up to now, dynamical switching from the individual PIT effect to the dual-band PIT effect has yet to be experimentally explored. However, the dynamic modulation of the PIT effect from an individual window to dual windows has great prospects for applications in multi-frequency filtering, multi-mode operation devices, and optical storage.

In this work, we numerically and experimentally study an ultrafast all-optical switching of the PIT effect in a metaphotonic device. A CW resonator and two pairs of SRRs are implemented in such a device to support the bright and dark modes that can destructively interfere with each other, resulting in the generation of the PIT phenomenon. In order to switch the individual PIT effect, we incorporate the long lateral metal bars (LLMBs) and silicon $(\mathrm{Si})$ islands into the $\mathrm{CW}$ resonator and SRRs. Additionally, taking advantage of the broken $\mathrm{C}_{2}$ structural symmetry, we impose a quasi-dark resonator in this device and observe a dual-band PIT effect under an optical excitation. Moreover, a rigorous numerical analysis of dual-band PIT windows is performed. According to the optical-pump THz-probe (OPTP) measurement, the ultrafast response time is verified to be in the sub-nanosecond scale.

\section{Experiments}

\subsection{Sample characterization}

The fabrication of a metamaterial device is processed as follows. A standard Si-on-sapphire (SOS) wafer was selected as the substrate. The SOS wafer consists of sapphire layer and Si layer with thicknesses of $525 \mu \mathrm{m}$ and $500 \mathrm{~nm}$, respectively. A photoresist layer was spun on the cleaned SOS wafer and drawn by a laser direct writing setup. After that, the photoresist layer was processed by soft bake. Then, the metal chromium (Cr) and gold $\mathrm{Au})$ were sputtered onto the SOS wafer in order as a coherent layer and metamaterial, respectively. The total height of the metal structure was about $150 \mathrm{~nm}$. After processing by the liftoff technique, the photoresist layer resolved and left an intact metamaterial device. The epitaxial $\mathrm{Si}$ was processed by dry etching.

\subsection{Numerical simulation}

The simulation of the $\mathrm{THz}$ probe on the metamaterial device is performed using the classical finite element method. The $\mathrm{THz}$ probe is set, transmitting normally to the surface of the metamaterial device, the electric-field intensity of which is set as $1 \mathrm{~V} / \mathrm{m}$. The permittivity of sapphire is set as 10.5 , from the comparison of $\mathrm{THz}$ pulse delay between the absence and presence of a sapphire in the passing way. The permittivity of $\mathrm{Si}$ is set as 11.7. In addition, the conductivity of $\mathrm{Au}$ is set as $7 \times 10^{6} \mathrm{~S} / \mathrm{m}$. The intensity of electric field $E$ is numerically calculated as $E=$ $\sqrt{E_{x}^{2}+E_{y}^{2}+E_{z}^{2}}$ from the surface of the metamaterial.

\subsection{Measurements in OPTP system}

As an active control sample of the $\mathrm{THz}$ metamaterial, we characterize the dynamic response using a commercial OPTP time-domain spectral system (TTT-02-OPTP, from TuoTuo Technology). After the Ti:sapphire amplifier, the output beam produces femtosecond pulses with a width of $\sim 100 \mathrm{fs}$, a repetition rate of $1 \mathrm{kHz}$, and a center wavelength of $800 \mathrm{~nm}$. The laser pulses are split by a half-mirror. Part of laser pulses pump the ZnTe crystal to generate $\mathrm{THz}$ radiation, which transmits through the metadevice at normal incidence. At the end of the optical path, another $\mathrm{ZnTe}$ crystal is used for detecting the $\mathrm{THz}$ signals transmitting through the metadevice. The other part of the laser pulses illuminate on the area of the metadevice that the $\mathrm{THz}$ pulses spot on [see the schematic profile in Fig. 1(a)]. The optical beam with a diameter of $\sim 5 \mathrm{~mm}$ spatially overlaps the $\mathrm{THz}$ beam containing a diameter of $\sim 2.2 \mathrm{~mm}$ at the surface of the metadevice, which ensures the homogenous optical excitation of Si over the area probed by the $\mathrm{THz}$ beam. A standard Fourier transform is used to recover the frequency-domain $\mathrm{THz}$ spectra from the time-domain $\mathrm{THz}$ waveforms. The meta-atom is composed of a CW resonator, an LLMB, and two pairs of SRRs, as presented in Fig. 1(b). Notably, in one pair of SRRs, $\mathrm{Si}$ islands are evaporated onto the gaps. The metaphotonic device is made via a standard micro- and nano-fabrication process, whose optical microscopic image is shown in Fig. 1(c).

\section{Results and Discussion}

Figure 2(a) shows the temporal evolution of the THz transmission spectra of the metadevice, at an optical pump power of

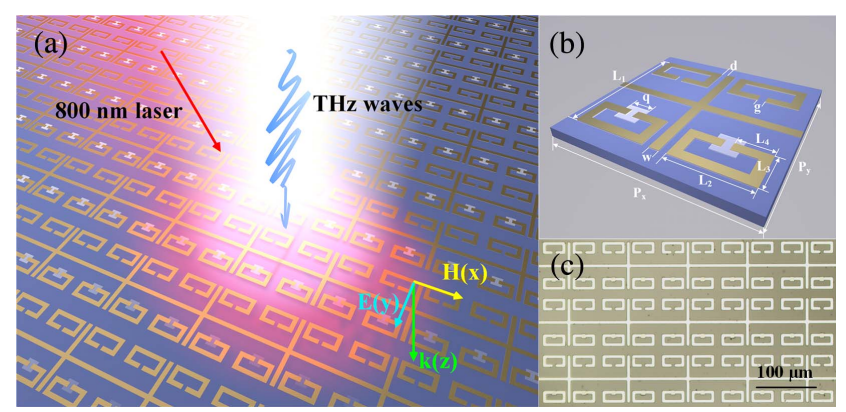

Fig. 1. (a) Schematic illustration of the metaphotonic device with triatomic structural molecules for OPTP spectroscopy. The metadevice is probed by $\mathrm{THz}$ beams at normal incidence and excited by an optical pump at oblique incidence. (b) Unit cell of the metamaterial comprising a CW resonator, an LLMB, and two pairs of SRRs. The geometrical parameters are as follows: $P_{x}=P_{y}=100, w=5, g=4, q=12, L_{1}=80, L_{2}=38, L_{3}=20$, and $L_{4}=17 \mu \mathrm{m}$. (c) Optical microscopic image of the metamaterials. 

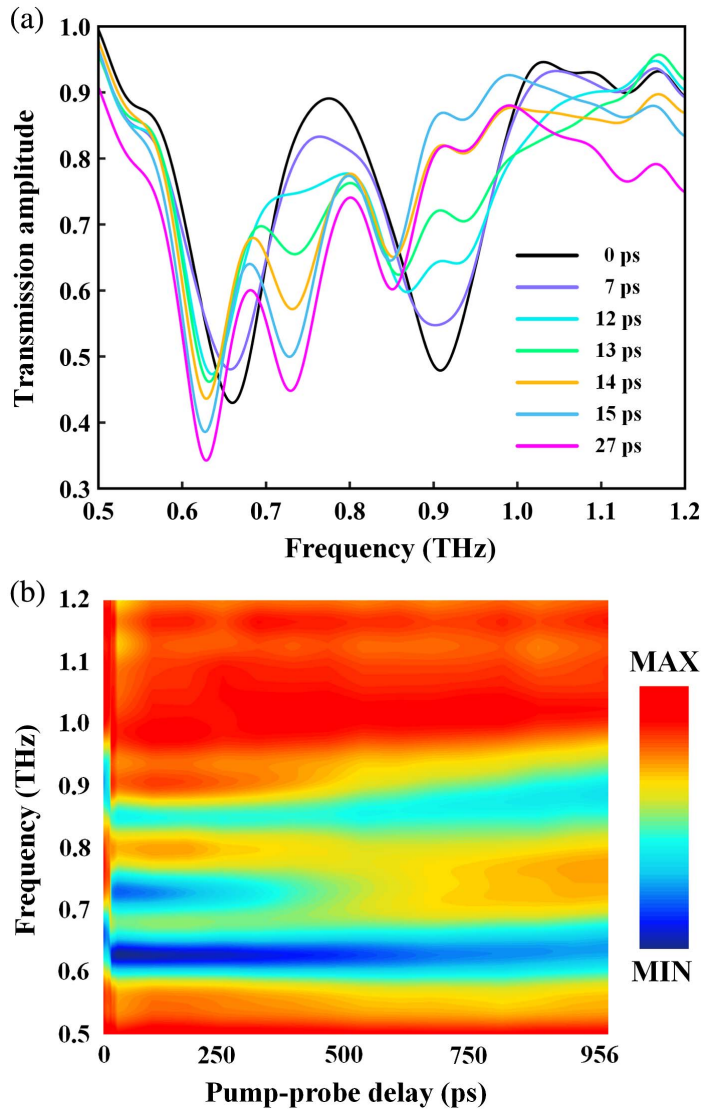

Fig. 2. (a) Experimentally measured THz transmission spectra of the metadevice at various pump-probe delays (as labeled). (b) Color map of THz transient transmissions against pump-probe delay and frequency.

$2500 \mathrm{~mW} / \mathrm{cm}^{2}$. The pump-probe delay represents the relative time delay between the arrivals of the $\mathrm{THz}$ probe pulse and laser pump pulse at the same region of the metadevice, which is controlled by moving the translational time delay stage. With a series of pump-probe delays from -4 ps to $956 \mathrm{ps}$, the transmitted $\mathrm{THz}$ probe pulses are measured and then transformed into a temporal evolution profile of transmission spectra. It is obvious that the appearance of the transmission dip at $0.73 \mathrm{THz}$ is faster than that of other transmission dips around because the quasidark resonance mode that induces the transmission dip is more sensitive to the free carriers in the Si island and optical pumps than the bright mode and dark mode. The coupling between the dark resonance mode and the bright resonance mode is modulated indirectly by the quasi-dark mode and not so sensitive to the optical pumps. Therefore, the temporal evolution of the transmission amplitude is attributed to the excitation and relaxation of carriers in $\mathrm{Si}$. The relaxation dynamics can be described by employing a mono-exponential decay equation, $-\frac{T(t)-T_{0}}{T_{0}}=$ $A_{0}+A_{1} \exp \left[\left(t-t_{0}\right) / \tau\right]$.

We proceed to explore the impact of pump power on the modulation of the PIT effect. Figure 3 illustrates the vivid comparison between the numerically calculated transmission spectra and the experimentally measured results at a pump-probe delay

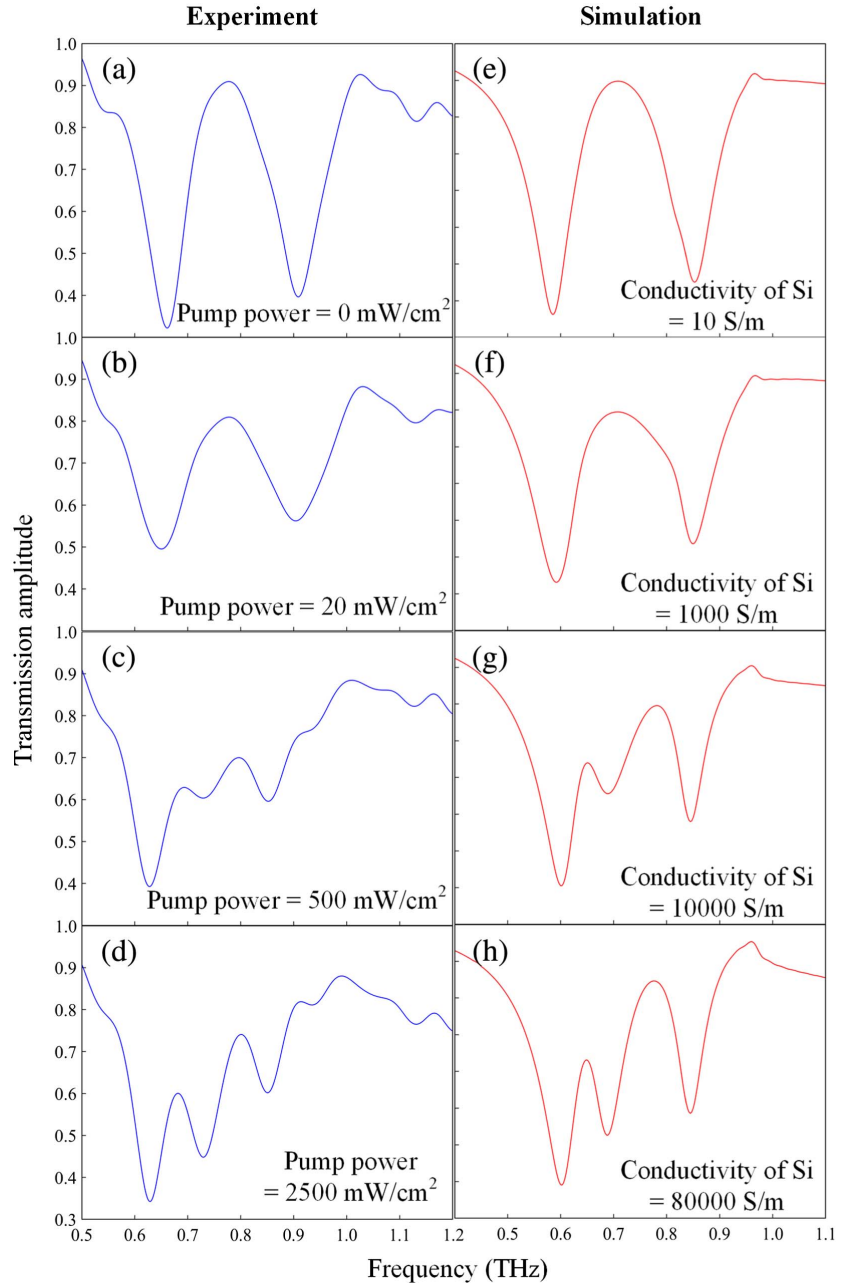

Fig. 3. (a)-(d) Spectral dispersion of measured THz transmission for the triatomic metamaterials with a series of selected pump power levels las labeled] at a fixed pump-probe delay of 6 ps for which the photo-induced change in spectral dispersion is maximized. (e)-(h) Corresponding numerically simulated transmission spectra varying with the conductivity of Si las labeled).

time of 6 ps. The blue curve in Fig. 3(a) represents a typical PIT effect that is characterized by a transparent window under a broad absorption background in the absence of a laser pump. With the increment of optical pump power, the PIT effect is gradually suppressed, since a large amount of free carriers are photoexcited into the conduction band, activating the quasidark resonance mode and shielding the destructive coupling between the dark resonance mode and bright resonance mode. At a pump power of $2500 \mathrm{~mW} / \mathrm{cm}^{2}$, the dual-band PIT effect occurs, as depicted in Fig. 3(d). One significant finding is that a dip is introduced at the transparent peak in Fig. 3(a), which is caused by the quasi-dark resonance mode. Figures $3(\mathrm{e})-3(\mathrm{~h})$ show the numerically simulated spectral dispersion, accounting for a series of Si conductivities, which coincide well with the experimental data. Alternatively, some subtle differences in resonant frequencies are discovered between the simulated and 
measured results, which may be caused by imperfections in sample preparation.

Due to the tunable dip in the PIT window, a remarkable variation of slow light effect could be controlled in our designed metadevice. The slow light effect is usually represented by a group delay, $\Delta t_{g}$, which describes the time delay of a $\mathrm{THz}$ wave passing through the metadevice compared with its transmission in air. This parameter is mathematically given by

$$
\Delta t_{g}=\frac{\mathrm{d} \varnothing}{\mathrm{d} \varphi}
$$

where $\varnothing$ is the phase of transmission through the metadevice. Its differential as a function of $\varphi$, which is the angular frequency of the $\mathrm{THz}$ wave, determines the group delay shown in Fig. 4. Precisely, Figs. 4(a)-4(e) show the calculated group delay of

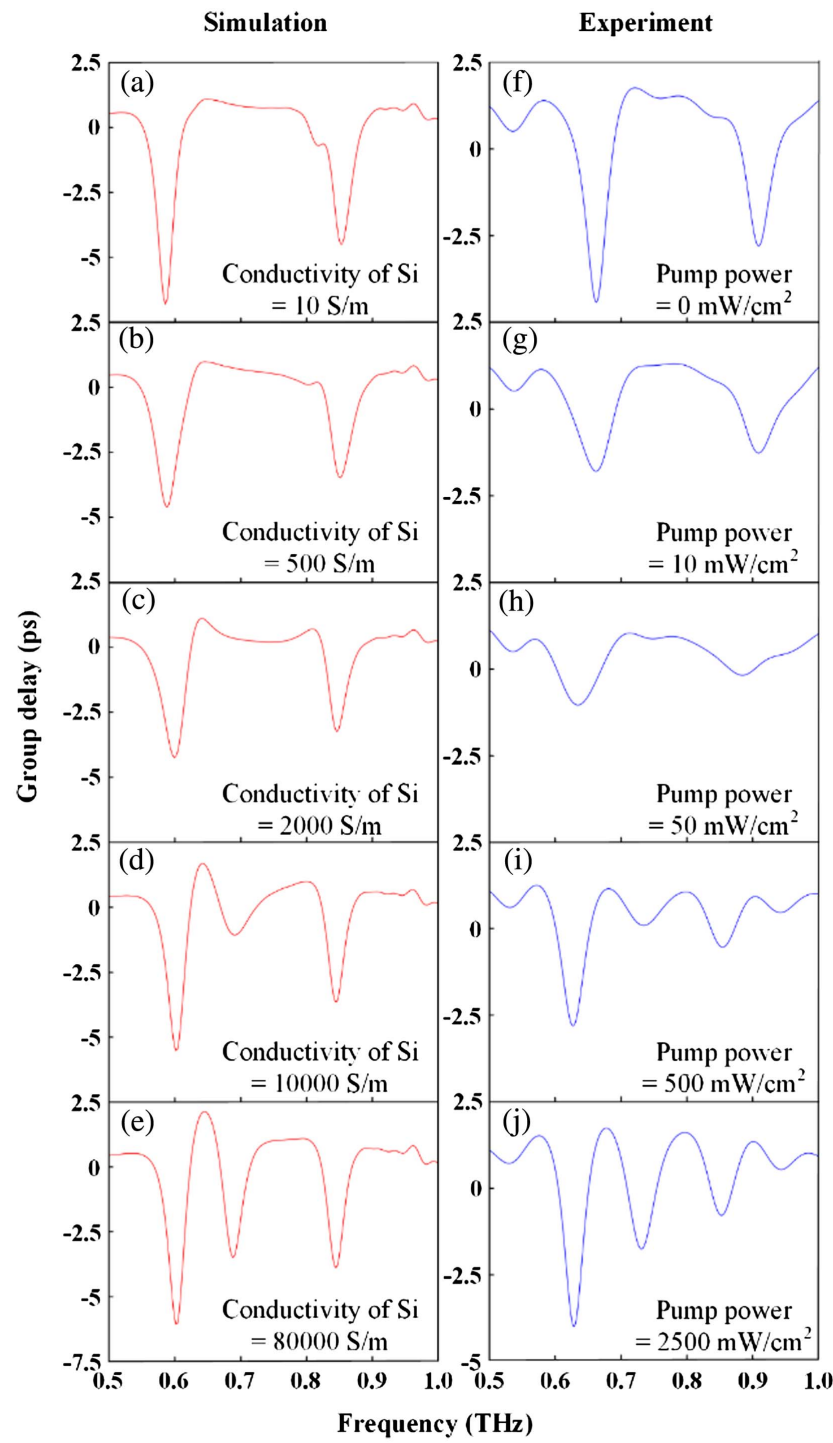

Fig. 4. (a)-(e) Numerically calculated and ( $f$ )-(j) experimentally measured group delays of the $\mathrm{THz}$ waves transmitted through the metadevice for a series of conductivity of Si and pump power (as labeled), respectively. the $\mathrm{THz}$ wave traveling through the metadevice at different $\mathrm{Si}$ conductivity. As expected, a remarkable switching behavior of group delay is observed, manifesting a strong phase dispersion. Meanwhile, a dip occurs in the group delay curve when the conductivity of Si increases, which reaches -3.5 ps at $80,000 \mathrm{~S} / \mathrm{m}$. The experimental results are depicted by Figs. 4(f)-4(j). In Fig. 4(j), a dip is found at $0.73 \mathrm{THz}$, which is caused by the confined electric quasi-dark mode oscillated in the metamaterials at the same frequency. However, the shape of dips in the calculated results is sharper than that in the experimental cases. A reasonable explanation is that the limited minimal step of translational time delay stage decreases the precision of the group delay spectrum.

To understand the evolution of the PIT effect in the triatomic metamaterial, we calculate the transmission response and electric-field profiles in CW resonators and triatomic metamaterials, with the results presented by Fig. 5. Figure 5(a) signifies the simulated transmission response of a single CW resonator. The localized surface plasmon (LSP) resonance is excited in CW resonators and leads to a transparent dip at $0.72 \mathrm{THz}$. By placing the SRR and LLMB around the CW resonator and across the CW resonator, respectively, we realize the PIT effect with an apparent transparent peak at $0.71 \mathrm{THz}$. The near-field coupling between CW resonators and SRRs excites the SRRs on both sides of CW resonators. As a result, the PIT effect comes from the destructive interference between the LSP resonance and the inductive-capacitive (LC) resonance, which are supported by the CW resonator and SRRs, respectively. Here, the CW resonators and SRRs act as the bright mode and dark mode, respectively. Figures 5(b) and 5(e) show the transmission spectrum and electric-field profiles. In order to accomplish a three-level coupling interaction in the triatomic metamaterials, we introduce the Si islands on a couple of splits in SRRs [shown in Fig. 1(b)]. By injecting laser pumps onto the Si islands, a couple of SRRs are short-circuited by photo-induced
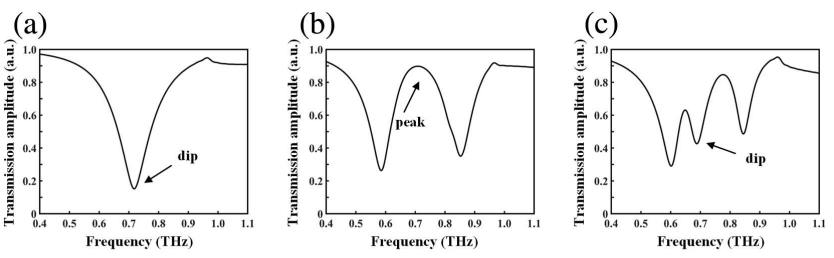

(d)

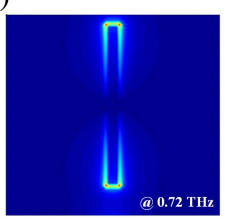

(e)

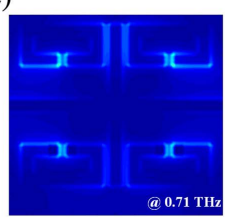

(f)

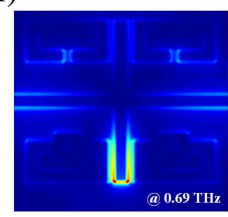

Fig. 5. Simulated THz transmission spectra through (a) CW resonators, triatomic metamaterials (b) in the dark and (c) pumped by laser pulses. The conductivity of $\mathrm{Si}$ is selected as $10 \mathrm{~S} / \mathrm{m}$ (in the dark) and $80,000 \mathrm{~S} / \mathrm{m}$ (under laser pump). (d)-(f) Corresponding electric-field profiles in the triatomic metamaterial. 


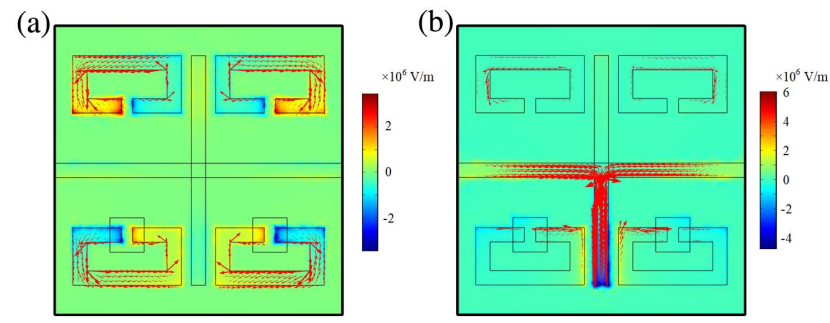

Fig. 6. Distribution of surface current density of metamaterials at resonance frequency. (a) Surface current at the transparent peak for $\sigma_{\mathrm{Si}}=10 \mathrm{~S} / \mathrm{m}$ (case without pump). (b) Surface current at the transparent dip for $\sigma_{\mathrm{Si}}=80,000 \mathrm{~S} / \mathrm{m}$ (case with the highest pump fluence).

carriers in Si. Therefore, the destructive interference between CW resonators and half of the SRRs is suppressed. For lack of local coupling between electric fields in CW resonators and short-circuited SRRs, another type of LSP resonance is generated between the LLMB and CW resonator, because of the asymmetry in the triatomic metamaterials. By simulating the distributions of surface current density at the peak of individual PIT effects and the dip of dual-band PIT effects, respectively, as shown in Fig. 6, we can intuitively understand the underlying physics in switching of the PIT effect. For the case without pump, the dark mode that is coupled with the bright mode occupies the main resonant mode [see Fig. 6(a)]. Nevertheless, when under optical pump irradiation, the quasi-dark mode is excited, whereas the surface current mainly focuses on the LLMB and CW resonator [see Fig. 6(b)]. Accompanied by the new LSP resonance, a transparent dip occurs at the original transparent peak, switching the PIT effect from individual window into dual windows, as exhibited in Fig. 5(c).

\section{Conclusion}

In summary, we have proposed a triatomic metamaterial device comprising CW resonators, SRRs, and LLMBs and hybridized with $\mathrm{Si}$ islands. An individual PIT effect occurs when $\mathrm{THz}$ waves transmit through the metaphotonic device, owing to the coupling effect between the bright mode and dark mode supported by CW resonators and SRRs. Excited by optical pumps, a pair of SRRs are short-circuited by photo-induced carriers in Si islands, promoting the occurrence of the quasi-dark mode supported by $\mathrm{CW}$ resonator and LLMB. Simultaneously, the switching of the PIT effect from individual transparent windows to dual-band windows is also achieved. A prominent slow light effect is measured during the modulating process. Benefiting from the ultrafast relaxation time of carriers in epitaxial $\mathrm{Si}$, the recovery time of the modulating process is in a nanosecond timescale.

\section{Acknowledgement}

This work was supported by the National Natural Science Foundation of China (Nos. 11804387, 11802339, 11805276, 11902358, 61805282, and 61801498), the Scientific Researches
Foundation of National University of Defense Technology (Nos. ZK18-03-22, ZK18-01-03, and ZK18-03-36), and the Science Fund for Distinguished Young Scholars of Hunan Province (No. 2020JJ2036).

${ }^{\dagger}$ These authors contributed equally to this work.

\section{References}

1. T.-T. Kim, H.-D. Kim, R. Zhao, S. S. Oh, T. Ha, D. S. Chung, Y. H. Lee, B. Min, and S. Zhang, "Electrically tunable slow light using graphene metamaterials," ACS Photon. 5, 1800 (2018).

2. H. Jung, H. Jo, W. Lee, B. Kim, H. Choi, M. S. Kang, and H. Lee, "Electrical control of electromagnetically induced transparency by terahertz metamaterial funneling," Adv. Opt. Mater. 7, 1801205 (2019).

3. K. M. Devi, M. Islam, D. R. Chowdhury, A. K. Sarma, and G. Kumar, "Plasmon-induced transparency in graphene-based terahertz metamaterials," EPL 120, 27005 (2017).

4. J. Gu, R. Singh, X. Liu, X. Zhang, Y. Ma, S. Zhang, S. A. Maier, Z. Tian, A. K. Azad, H.-T. Chen, A. J. Taylor, J. Han, and W. Zhang, "Active control of electromagnetically induced transparency analogue in terahertz metamaterials," Nat. Commun. 3, 1151 (2012).

5. A. Ahmadivand, B. Gerislioglu, and Z. Ramezani, "Gated graphene islandenabled tunable charge transfer plasmon terahertz metamodulator," Nanoscale 11, 8091 (2019).

6. M. Manjappa, Y. K. Srivastava, A. Solanki, A. Kumar, T. C. Sum, and R. Singh, "Hybrid lead halide perovskites for ultrasensitive photoactive switching in terahertz metamaterial devices," Adv. Mater. 29, 1605881 (2017).

7. P. Pitchappa, A. Kumar, S. Prakash, H. Jani, T. Venkatesan, and R. Singh, "Chalcogenide phase change material for active terahertz photonics," Adv. Mater. 31, 1808157 (2019).

8. H.-T. Chen, J. F. O'Hara, A. K. Azad, A. J. Taylor, R. D. Averitt, D. B. Shrekenhamer, and W. J. Padilla, "Experimental demonstration of frequency-agile terahertz metamaterials," Nat. Photon. 2, 295 (2008).

9. N.-H. Shen, M. Massaouti, M. Gokkavas, J.-M. Manceau, E. Ozbay, M. Kafesaki, T. Koschny, S. Tzortzakis, and C. M. Soukoulis, "Optically implemented broadband blueshift switch in the terahertz regime," Phys. Rev. Lett. 106, 037403 (2011).

10. Z. Chen, X. Chen, L. Tao, K. Chen, M. Long, X. Liu, K. Yan, R. I. Stantchev, E. Pickwell-MacPherson, and J.-B. Xu, "Graphene controlled Brewster angle device for ultra broadband terahertz modulation," Nat. Commun. 9, 4909 (2018).

11. M. D. Goldflam, M. K. Liu, B. C. Chapler, H. T. Stinson, A. J. Sternbach, A. S. McLeod, J. D. Zhang, K. Geng, M. Royal, B.-J. Kim, R. D. Averitt, N. M. Jokerst, D. R. Smith, H.-T. Kim, and D. N. Basov, "Voltage switching of a $\mathrm{VO}_{2}$ memory metasurface using ionic gel," Appl. Phys. Lett. 105, 041117 (2014).

12. H. Jung, J. Koo, E. Heo, B. Cho, C. In, W. Lee, H. Jo, J. H. Cho, H. Choi, M. S. Kang, and H. Lee, "Electrically controllable molecularization of terahertz meta-atoms," Adv. Mater. 30, 1802760 (2018).

13. Y. Hu, T. Jiang, J. Zhou, H. Hao, H. Sun, H. Ouyang, M. Tong, Y. Tang, H. Li, J. You, X. Zheng, Z. Xu, and X. Cheng, "Ultrafast terahertz transmission/ group delay switching in photoactive $\mathrm{WSe}_{2}$-functionalized metaphotonic devices," Nano Energy 68, 104280 (2020).

14. J. Zhou, Y. Hu, T. Jiang, H. Ouyang, H. Li, Y. Sui, H. Hao, J. You, X. Zheng, $\mathrm{Z}$. $\mathrm{Xu}$, and $\mathrm{X}$. Cheng, "Ultrasensitive polarization-dependent terahertz modulation in hybrid perovskites plasmon-induced transparency devices," Photon. Res. 7, 994 (2019).

15. J. Ji, S. Zhou, W. Wang, F. Ling, and J. Yao, "Active control of terahertz plasmon-induced transparency in the hybrid metamaterial/monolayer $\mathrm{MoS}_{2} / \mathrm{Si}$ structure," Nanoscale 11, 9429 (2019).

16. P. Pitchappa, M. Manjappa, C. P. Ho, R. Singh, N. Singh, and C. Lee, "Active control of electromagnetically induced transparency analog in terahertz MEMS metamaterial," Adv. Opt. Mater. 4, 541 (2016). 
17. C. Liu, P. Liu, C. Yang, Y. Lin, and H. Liu, "Analogue of dual-controlled electromagnetically induced transparency based on a graphene metamaterial," Carbon 142, 354 (2019).

18. S. J. Kindness, N. W. Almond, B. Wei, R. Wallis, W. Michailow, V. S. Kamboj, P. Braeuninger-Weimer, S. Hofmann, H. E. Beere, D. A. Ritchie, and R. Degl'Innocenti, "Active control of electromagnetically induced transparency in a terahertz metamaterial array with graphene for continuous resonance frequency tuning," Adv. Opt. Mater. 6, 1800570 (2018).

19. Y. Wu, C. La-o-vorakiat, X. Qiu, J. Liu, P. Deorani, K. Banerjee, J. Son, Y. Chen, E. E. M. Chia, and H. Yang, "Graphene terahertz modulators by ionic liquid gating," Adv. Mater. 27, 1874 (2015).

20. W. Y. Kim, H.-D. Kim, T.-T. Kim, H.-S. Park, K. Lee, H. J. Choi, S. H. Lee, J. Son, N. Park, and B. Min, "Graphene-ferroelectric metadevices for nonvolatile memory and reconfigurable logic-gate operations," Nat. Commun. 7, 10429 (2016).

21. Z. Xu and Y.-S. Lin, "A stretchable terahertz parabolic-shaped metamaterial," Adv. Opt. Mater. 7, 1900379 (2019).

22. J. Ji, S. Zhou, W. Wang, C. Luo, Y. Liu, F. Ling, and J. Yao, "Active multifunctional terahertz modulator based on plasmonic metasurface," Opt. Express 27, 2363 (2019).

23. S. H. Lee, M. Choi, T.-T. Kim, S. Lee, M. Liu, X. Yin, H. K. Choi, S. S. Lee, C.-G. Choi, S.-Y. Choi, X. Zhang, and B. Min, "Switching terahertz waves with gate-controlled active graphene metamaterials," Nat. Mater. 11, 936 (2012).

24. M. M. Jadidi, A. B. Sushkov, R. L. Myers-Ward, A. K. Boyd, K. M. Daniels, D. K. Gaskill, M. S. Fuhrer, H. D. Drew, and T. E. Murphy, "Tunable terahertz hybrid metal-graphene plasmons," Nano Lett. 15, 7099 (2015).

25. S. Xiao, T. Wang, T. Liu, C. Zhou, X. Jiang, and J. Zhang, "Active metamaterials and metadevices: a review," J. Phys. D: Appl. Phys. 53, 503002 (2020).

26. P. Tassin, L. Zhang, T. Koschny, E. N. Economou, and C. M. Soukoulis, "Low-loss metamaterials based on classical electromagnetically induced transparency,” Phys. Rev. Lett. 102, 053901 (2009).

27. Q. Li, Z. Tian, X. Zhang, N. Xu, R. Singh, J. Gu, P. Lv, L.-B. Luo, S. Zhang, J. Han, and W. Zhang, "Dual control of active graphene-silicon hybrid metamaterial devices," Carbon 90, 146 (2015).

28. S. Zhang, D. A. Genov, Y. Wang, M. Liu, and X. Zhang, "Plasmon-induced transparency in metamaterials," Phys. Rev. Lett. 101, 047401 (2008).

29. H. Sun, Y. Hu, Y. Tang, J. You, J. Zhou, H. Liu, and X. Zheng, "Ultrafast polarization-dependent all-optical switching of germanium-based metaphotonic devices," Photon. Res. 8, 263 (2020).

30. H. Sun, J. Yang, H. Liu, D. Wu, and X. Zheng, "Process-controllable modulation of plasmon-induced transparency in terahertz metamaterials," Chin. Opt. Lett. 19, 013602 (2021).

31. H. Sun, Y. Tang, Y. Hu, J. You, H. Liu, and X. Zheng, "Active formatting modulation of electromagnetically induced transparency in metamaterials," Chin. Opt. Lett. 18, 092402 (2020).

32. Y. Hu, T. Jiang, J. Zhou, H. Hao, H. Sun, H. Ouyang, M. Tong, Y. Tang, H. Li, J. You, X. Zheng, Z. Xu, and X. Cheng, "Ultrafast terahertz frequency and phase tuning by all-optical molecularization of metasurfaces," Adv. Opt. Mater. 7, 1901050 (2019).

33. J. Zhou, C. Zhang, Q. Liu, J. You, X. Zheng, X. Cheng, and T. Jiang, "Controllable all-optical modulation speed in hybrid silicon-germanium devices utilizing the electromagnetically induced transparency effect," Nanophotonics 9, 2797 (2020).

34. Y. Hu, T. Jiang, H. Sun, M. Tong, J. You, X. Zheng, Z. Xu, and X. Cheng, "Ultrafast frequency shift of electromagnetically induced transparency in terahertz metaphotonic devices," Laser Photon. Rev. 14, 1900338 (2020).

35. Y. Hu, J. You, M. Tong, X. Zheng, Z. Xu, X. Cheng, and T. Jiang, "Pump-color selective control of ultrafast all-optical switching dynamics in metaphotonic devices," Adv. Sci. 7, 2000799 (2020).

36. R. Sarkar, D. Ghindani, K. M. Devi, S. S. Prabhu, A. Ahmad, and G. Kumar, "Independently tunable electromagnetically induced transparency effect and dispersion in a multi-band terahertz metamaterial," Sci. Rep. 9, 18068 (2019).

37. J. Ding, B. Arigong, H. Ren, M. Zhou, J. Shao, M. Lu, Y. Chai, Y. Lin, and H. Zhang, "Tuneable complementary metamaterial structures based on graphene for single and multiple transparency windows," Sci. Rep. 4, 6128 (2014).

38. Y. Wang, M. Tao, Z. Pei, X. Yu, B. Wang, J. Jiang, and X. He, "Tunable bandwidth of double electromagnetic induced transparency windows in terahertz graphene metamaterial," RSC Adv. 8, 37057 (2018).

39. K. Zhang, C. Wang, L. Qin, R.-W. Peng, D.-H. Xu, X. Xiong, and M. Wang, "Dual-mode electromagnetically induced transparency and slow light in a terahertz metamaterial," Opt. Lett. 39, 3539 (2014).

40. F. Bagci and B. Akaoglu, "Single and multi-band electromagnetically induced transparency-like effects with a four-fold symmetric metamaterial design," Mater. Res. Express 6, 055806 (2019).

41. K. M. Devi, D. R. Chowdhury, G. Kumar, and A. K. Sarma, "Dual-band electromagnetically induced transparency effect in a concentrically coupled asymmetric terahertz metamaterial," J. Appl. Phys. 124, 063106 (2018).

42. C. Sun, J. Si, Z. Dong, and X. Deng, "Tunable multispectral plasmon induced transparency based on graphene metamaterials," Opt. Express 24, 11466 (2016).

43. S. Hu, H. Yang, S. Han, X. Huang, and B. Xiao, "Tailoring dual-band electromagnetically induced transparency in planar metamaterials," J. Appl. Phys. 117, 043107 (2015).

44. C. Tang, Q. Niu, B.-X. Wang, and W.-Q. Huang, "Design of dual-band plasmon-induced transparent effect based on composite structure of closed-ring and square patch," Plasmonics 14, 533 (2019).

45. Z. Dong, C. Sun, J. Si, and X. Deng, "Tunable polarization-independent plasmonically induced transparency based on metal-graphene metasurface," Opt. Express 25, 12251 (2017).

46. J. Kim, R. Soref, and W. R. Buchwald, "Multi-peak electromagnetically induced transparency (EIT)-like transmission from bull's-eye-shaped metamaterial," Opt. Express 18, 17997 (2010). 